\title{
A contribuição do potencial terapêutico da ioga em tempos de COVID-19
}

\author{
The contribution of the therapeutic potential of yoga in COVID-19 times \\ La contribución del potencial terapéutico del yoga en tiempos de COVID-19
}

\author{
Narottam Sócrates Garcia CHUMPITAZ ${ }^{(1)}$ \\ Emille Magalhães Neves CAMPOS ${ }^{(1)}$ \\ Jhon Andreo Almeida dos SANTOS ${ }^{(1)}$ \\ Giovanni de Souza MOTA ${ }^{(1)}$ \\ Francisco Carlos Carneiro da SILVA ${ }^{(2)}$ \\ Karine Kersting PULS ${ }^{(3)}$
}

Recebido: 20 jul 2020

Revisado: 30 ago 2020

Aceito: 12 out 2020

Autor de correspondência: Narottam Sócrates Garcia Chumpitaz

narottamgarcia@gmail.com

Conflito de interesses:

Os autores declaram não haver nenhum interesse profissional ou pessoal que possa gerar conflito de interesses em relação a este manuscrito.

\footnotetext{
(1) Universidade Federal de Roraima - UFRR, Curso de Medicina, Boa Vista, RR, Brasil.

(2) Universidade Federal de Roraima - UFRR, Curso de Medicina, Boa Vista, RR, Brasil.

(3)Universidade de Caxias do Sul - UCS, Curso de Medicina, Campus-Sede, Caxias do Sul, RS, Brasil.
}

\section{Resumo}

A pandemia provocada pelas infecções por coronavírus trouxe uma nova realidade e perspectiva de vida à humanidade. $O$ isolamento social, nunca estendido por tanto tempo e simultaneamente por todo o mundo, tornou-se essencial para evitar a superlotação da rede pública de saúde, a qual já é colapsada pelo crescente número de hospitalizações. Proveniente dessa miríade de acontecimentos contemporâneos, originou-se uma problemática inesperada adjacente à pandemia: a comunidade científica mundial está focada, predominantemente, no patógeno SARS-CoV-2 e em seu risco biológico, num esforço para compreender os mecanismos fisiopatológicos envolvidos e estagnar a disseminação da doença. Em razão disso, a saúde mental individual foi posicionada em um plano secundário, principalmente em relação aos meios como cada indivíduo enfrenta o distanciamento social. Não obstante, neste texto, busca-se fomentar e divulgar o amplo efeito terapêutico da ioga em tempos de COVID-19 sobre a saúde mental, que favorece a conexão mente-corpo, o qual desencadeia reações fisiológicas notáveis, por exemplo, a liberação de neurotransmissores de relaxamento e prazer, a ação no eixo hipotalâmicopituitário-adrenal e liberação de fatores neurotróficos. Intrínseca a essa atividade, estão o relaxamento das emoções, o controle do humor, e o autoconhecimento, ao passo que todos esses aspectos trabalham tanto funções físicas, quanto psicológicas. $O$ principal objetivo deste trabalho é o conhecimento de tal terapia potencialmente benéfica, pois além de ser uma alternativa acessível para prática individual, é um alicerce para o alcance de uma melhor condição física e mental em tempos de COVID-19.

Descritores: Ioga; Saúde Mental; Isolamento Social; Infecções por Coronavírus. 


\title{
Abstract
}

The pandemic caused by coronavirus infections brought a new reality and perspective on life for humanity. Social isolation, never before extended for so long and simultaneously all over the world, has become essential to avoid overcrowding in the public health network, which is already collapsed by the growing number of hospitalizations. From this myriad of contemporary events, an unexpected problem has arisen adjacent to the pandemic: the world scientific community is predominantly focused on the SARS-CoV-2 pathogen and its biological risk, in an effort to understand the pathophysiological mechanisms involved and stagnate the spread of the disease. As a result, individual mental health was placed on a secondary level, mainly in relation to the means by which each individual faces social distance. Nevertheless, this text seeks to promote and disseminate the broad therapeutic effect of yoga in COVID-19 times on mental health, which favors the mind-body connection, which triggers notable physiological reactions, for example, the release of neurotransmitters for relaxation and pleasure, action on the hypothalamic-pituitary-adrenal axis and release of neurotrophic factors. Intrinsic to this activity are relaxation of emotions, the control of mood, and self-awareness, while working with both physical and psychological functions. The main objective of this work is the knowledge of such a potentially beneficial therapy, because besides being an accessible alternative for individual practice, it is a foundation for achieving a better physical and mental condition in times of COVID-19.

Keywords: Yoga; Mental Health; Social Isolation; Coronavirus Infections.

\begin{abstract}
Resumen
La pandemia causada por las infecciones por coronavirus trajo una nueva realidad y perspectiva de vida para la humanidad. El aislamiento social, nunca extendido por tanto tiempo y simultáneamente en todo el mundo, se ha vuelto fundamental para evitar el hacinamiento en la red de salud pública, que ya está colapsada por el creciente número de hospitalizaciones. A partir de esta miríada de eventos contemporáneos, surgió un problema inesperado junto a la pandemia: la comunidad científica mundial se centra predominantemente en el patógeno SARS-CoV-2 y su riesgo biológico, en un esfuerzo por comprender los mecanismos fisiopatológicos involucrados y estancarse. propagación de la enfermedad. Como resultado, la salud mental individual se colocó en un nivel secundario, principalmente en relación con los medios por los cuales cada individuo enfrenta la distancia social. Sin embargo, en este texto buscamos promover y dar a conocer el amplio efecto terapéutico del yoga en tiempos de COVID-19 sobre la salud mental, que favorece la conexión mentecuerpo, lo que desencadena reacciones fisiológicas notables, por ejemplo, la liberación de neurotransmisores de relajación y placer, acción sobre el eje hipotalámico-pituitario-adrenal y liberación de factores neurotróficos. Intrínsecos a esta actividad, están la relajación de las emociones, el control del estado de ánimo y el autoconocimiento, mientras que todos estos aspectos trabajan tanto en funciones físicas como psicológicas. El principal objetivo de este trabajo es el conocimiento de una terapia tan potencialmente beneficiosa, pues además de ser una alternativa accesible para la práctica individual, es una base para lograr una mejor condición física y mental en tiempos de COVID-19. Palabras-claves: Yoga; Salud Mental; Aislamiento Social; Infecciones por Coronavirus.
\end{abstract}

\section{Introdução}

É notório que a infecção de coronavírus (COVID-19) disseminou-se rapidamente por todo o mundo, e a Organização Mundial de Saúde - OMS afirma que o grau de ansiedade e a preocupação aumentaram consideravelmente na população em geral e em determinados grupos. Isso pode ser observado, particularmente em idosos, em prestadores de cuidados de saúde e em pessoas com comorbidades associadas. O medo de contrair o vírus durante a pandemia impôs mudanças significativas na vida individual diária, como o estresse causado pelo risco iminente de infecção e prejuízos econômicos. Além disso, à medida que novas regras, como isolamento social, foram introduzidas, houve um aumento 
considerável nos níveis de solidão, depressão, uso nocivo de álcool e drogas, além do desenvolvimento comportamental de autoagressão ou de suicídio. ${ }^{1}$

Adentrando nessa temática, é importante lembrar que não é a primeira vez que a sociedade experimenta emergências de agentes invisíveis. Mesmo após duas epidemias de coronavírus no século XXI, cujas etiologias foram o SARS-CoV, em 2002, e o MERS-CoV, em 2012, com importantes impactos políticos, econômicos e psicossociais, observou-se que a população aprendeu pouco e não estava preparada para lidar com os problemas e desafios impostos pelo novo advento. ${ }^{2,3}$

Em meio a esse cenário, o recém-identificado causador (SARS-CoV-2) foi primeiramente observado em Wuhan, China, em 31 de dezembro de 2019. Em um mês, os casos documentados superaram aqueles da epidemia do coronavírus tipo-1, causador da Síndrome Aguda Respiratória Severa - SARS-CoV. A miríade de fatores influenciadores da pandemia da COVID-19 se evidencia tanto devido às características intrínsecas virais, como pelas condições sociodemográficas locais, a exemplo da alta densidade populacional, condições insalubres e inadequada infraestrutura do sistema de saúde. ${ }^{4} \mathrm{~A}$ alta virulência, observada por meio de 3 vias de transmissão (gotículas, contato de mucosa e aerossol), assim como o período de incubação prolongado (de até 14 dias), contribuíram para o rápido cenário disseminador viral, no qual a população vulnerável é representada principalmente por idosos, imunossuprimidos, pessoas com comorbidades crônicas e com histórico cirúrgico. ${ }^{5} \mathrm{O}$ seu longo e discreto período de incubação também possibilita a livre circulação de pessoas assintomáticas em espaços públicos, assim como realizar viagens sem a ciência de sua possível infecção. Portanto, a globalização também se classifica como um fator de expansão da COVID-19. ${ }^{2}$

Devido à urgência de compreender e reverter essa pandemia, a comunidade científica mundial está focando, preferencialmente, no patógeno e no seu risco biológico, objetivando compreender os mecanismos fisiopatológicos envolvidos, a fim de estagnar a disseminação da doença. Subsequentemente, a saúde mental da população foi colocada em um nível secundário de importância, principalmente no que diz respeito às maneiras pelas quais cada indivíduo enfrenta o distanciamento social. Nesse cenário, dispõem-se, em alcance midiático amplo, informações duvidosas ou até falsas sobre os fatores relacionados à transmissão viral, ao período de incubação, ao seu alcance geográfico, ao número de infectados e ao índice de mortalidade atual. Tudo isso acaba incitando a insegurança e o medo na população, gerando consequências em inúmeros setores, com implicações diretas para a vida diária da população e sua saúde mental. ${ }^{2}$

Corroborando esses determinantes, foi constatado que pacientes infectados com COVID-19 (ou com suspeita de infecção) podem sofrer intensas reações emocionais e 
comportamentais, como medo, tédio, solidão, ansiedade, insônia ou raiva, como já foi relatado sobre situações semelhantes no passado. ${ }^{6}$ Tais condições podem incorrer em surtos psicóticos, ou mesmo evoluir para transtorno de ansiedade generalizada e transtorno depressivo maior, podendo resultar em intenção suicida. ${ }^{7}$

Nesse viés, a condição mental dos indivíduos, segundo o artigo "Coping with coronavirus anxiety" de John Sharp, ${ }^{8}$ depende de escolhas saudáveis e razoáveis, a citar, o uso de estratégias calmantes. Neste panorama, as contribuições terapêuticas da prática de ioga podem auxiliar no relaxamento e no desenvolvimento de um bem-estar físico e mental durante o isolamento social.

Vale ressaltar que a ioga é uma das 29 Práticas Integrativas e Complementares - PICs, ofertadas pelo Sistema Único de Saúde do Brasil - SUS, o qual tem como um dos objetivos levar práticas corporais e meditativas à atenção primária, promovendo, principalmente, a promoção de saúde no SUS. ${ }^{9}$ Estas PICs compreendem não somente tradições de diferentes culturas, como a medicina tradicional chinesa, mas também abrangem práticas complementares pouco debatidas e exploradas, que não estão totalmente incorporadas ao SUS. Apesar de que, atualmente, dentro das PICs, as práticas corporativas e meditativas são as mais disseminadas na atenção primária. ${ }^{10}$

No estado do Rio Grande do Sul (RS), através da Nota Técnica 02/2018 - Yoga na rede de atenção à saúde, ${ }^{11}$ promovida pela Política Estadual de Práticas Integrativas e Complementares, objetiva-se orientar os gestores do SUS sobre a implantação da Yoga como PICs na Rede de Atenção à Saúde no RS.

Por meio deste texto, busca-se elucidar as contribuições terapêuticas da ioga frente à pandemia da COVID-19, pela ênfase em seus efeitos psicofisiológicos e em sua utilização como meio de manutenção da saúde mental.

\section{Ioga: história e impactos atuais}

A ioga é uma tradição milenar originária da Índia, a qual utiliza um conjunto de práticas psicofísicas, que implicam o autoconhecimento do ser, levando-o, ainda, a descobrir como ampliar os seus limites. Além disso, proporciona a capacidade de relaxar consigo mesmo, possibilitando que o indivíduo se sinta completo, integrado e em harmonia. ${ }^{12,13}$

A ioga, incluindo a meditação, podem ser práticas simples e úteis em casa, para a prevenção e gerenciamento pós-recuperação da COVID-19, de modo que estas terapias reduzem marcadores de inflamação e exercem considerável influência sobre muitos processos fisiológicos no organismo humano. ${ }^{14}$ Dentre eles, destacam-se os seus efeitos imunomoduladores, estes, embora incompletos, são demonstrados em estudos 
metodologicamente mais rigorosos para determinar as suas implicações clínicas para os resultados de doenças inflamatórias e infecciosas. ${ }^{15}$

Nesse viés, orientar-se por medidas sensatas, provenientes de especialistas em saúde pública, é a alternativa mais prudente nesse período. É nesse cenário que a ioga se insere, não somente como investimento cultural, mas também como atividade de integralização corpo-mente, de importante influência na estabilização da saúde mental durante a pandemia.

Em um estudo, ${ }^{16}$ cujo objetivo foi discutir a inserção das Práticas Integrativas e Complementares Grupais - PICs Grupais, nos serviços de saúde da Atenção Básica, das Unidades Básicas de Saúde da Família de um município nordestino, foram apresentadas as diversas modalidades e características das PICs Grupais (como as categorias de arte e cultura; grupos terapêuticos e de ajuda mútua; dança e outros). Destas, a ioga se configurou na categoria de "intervenção mente-corpo". ${ }^{16}$

Foi constatado que as PICs Grupais se figuram como um método para gerenciar as relações de poder vigentes na sociedade, em especial na área da saúde pública, pois levam a possibilidade de problematização acerca da garantia da integralidade, numa perspectiva dialógica, emancipadora, participativa e criativa, que articula saberes, práticas, vivências e espaços de saúde. Além disso, é afirmado que são potencialmente contribuintes para a desmedicalização nos serviços de saúde, reduzindo a sua mercantilização, uma vez que enriquecem a experiência do processo saúde/cuidado, por parte das gestões em saúde. ${ }^{16}$

\section{Efeitos psicofisiológicos da ioga em diferentes níveis de saúde mental}

É importante salientar que os estados mentais negativos não refletem somente o contexto externo no qual o indivíduo possa estar inserido, mas também as alterações fisiológicas originadas de transtornos mentais adjacentes, no caso de pacientes psiquiátricos, e os efeitos colaterais das medicações administradas para o seu tratamento. Nesse contexto, a resistência aos fármacos é um problema crescente e há milhões de pacientes pelo mundo cuja depressão, ansiedade ou esquizofrenia não são completamente resolvidas, apesar dos múltiplos experimentos com agentes psicofarmacológicos. Esse cenário leva pacientes e médicos a se esforçarem para equilibrar a relação de custobenefício, ou seja, a eficácia do medicamento contra o seu valor e efeitos colaterais, fato que frequentemente resulta na baixa adesão medicamentosa e ao relapso. ${ }^{17}$

Esse quadro pode ser exemplificado por uma análise retrospectiva, através da qual Keyloun e companheiros ${ }^{18}$ analisaram por 1 ano a aderência e persistência dos pacientes a 3 fatores: a medicações antidepressivas específicas, às classes terapêuticas e à terapia 
antidepressiva geral em múltiplos períodos (3, 6, 9 e 12 meses). Pela comparação, constatouse que ambos os critérios (aderência e persistência) diminuíram significativamente durante o ano de terapia para cada um dos 3 fatores, ao passo que nos primeiros 3 meses a taxa de aderência à terapia antidepressiva geral foi de 44\%, e ao final dos 12 meses, reduziu para $26 \%$. Já em relação a persistência, atingiram-se os valores de $48 \%$ no início e $22 \%$ ao final do período estudado. ${ }^{18}$

Isso demonstra que somente o uso de medicamentos não é suficiente para se atingir níveis plenos de saúde mental, haja vista a ampla variedade de fatores biopsicossociais que influenciam na vida humana. A demanda por modalidades de tratamento não farmacológico, dessa forma, exibe o enorme potencial terapêutico da ioga.

\section{Efeitos biofisiológicos da prática de ioga}

Dentre os múltiplos efeitos benéficos constatáveis com a prática de ioga, encontramse a sua influência em neurotransmissores, inflamação, estresse oxidativo, nível lipídico, fatores de crescimento e mensageiros secundários, em uma maneira amplamente similar a qual foi demonstrada com antidepressivos e psicoterapia, como esquematizado na Figura 1.

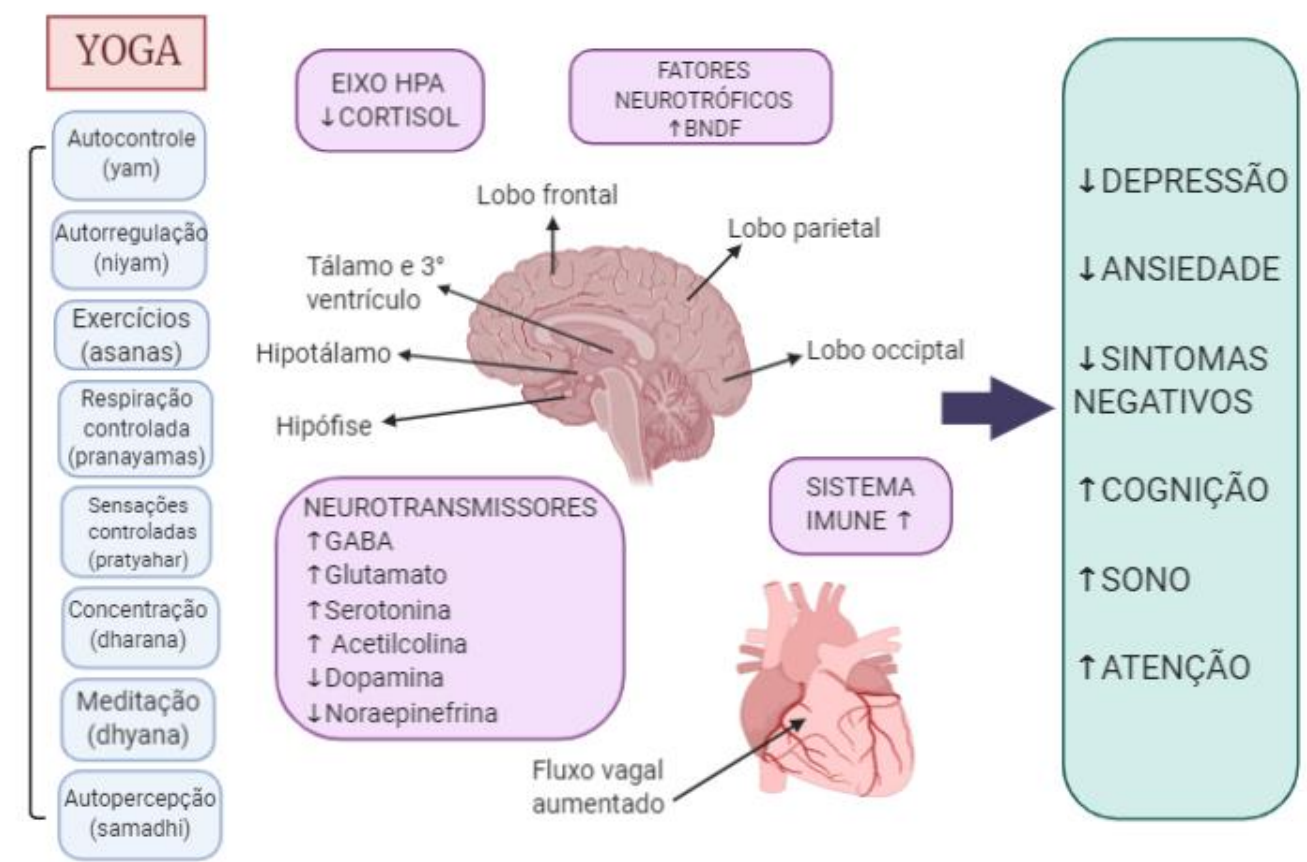

Figura 1. Efeitos psicofisiológicos da prática de ioga Fonte: Balasubramaniam, Telles, Doraiswamy, 2013.17, p. 3

Além disso, por meio dos efeitos de combinação de posturas físicas, a ioga e a meditação exercem influência sobre alterações de humor, responsáveis pelo aumento dos 
níveis do fator neurotrófico derivado do cérebro (BDNF), ${ }^{19}$ mediador da diferenciação, maturação e sobrevivência dos neurônios no sistema nervoso. Essa proteína assume também função neuroprotetora sob condições adversas, como estimulação glutamatérgica, isquemia cerebral, hipoglicemia e neurotoxicidade. Níveis rebaixados de BDNF são associados a doenças neurodegenerativas com perda neuronal, como Doença de Parkinson, Doença de Alzheimer, Esclerose Múltipla e Doença de Huntington. Outrossim, o BDNF pode ser útil na prevenção e manejo de doenças crônicas, destacando-se Diabetes Mellitus. ${ }^{17,19}$

Outros processos fisiológicos proporcionados pela ioga são aumento do tônus vagal, regulação negativa do eixo hipotalâmico-pituitário-adrenal e aumento dos níveis de ácido gama-aminobutírico (GABA), fato que implica a redução dos sintomas de Transtorno Depressivo Maior - TDM, como demonstrado na Figura 2. Essas observações coincidem com a teoria de que a estimulação vagal periférica e as terapias baseadas em ioga efetivamente elevam a atividade do sistema nervoso parassimpático (SNP) e consequentemente, os níveis de GABA, reduzindo a sintomatologia de tais doenças. ${ }^{20}$

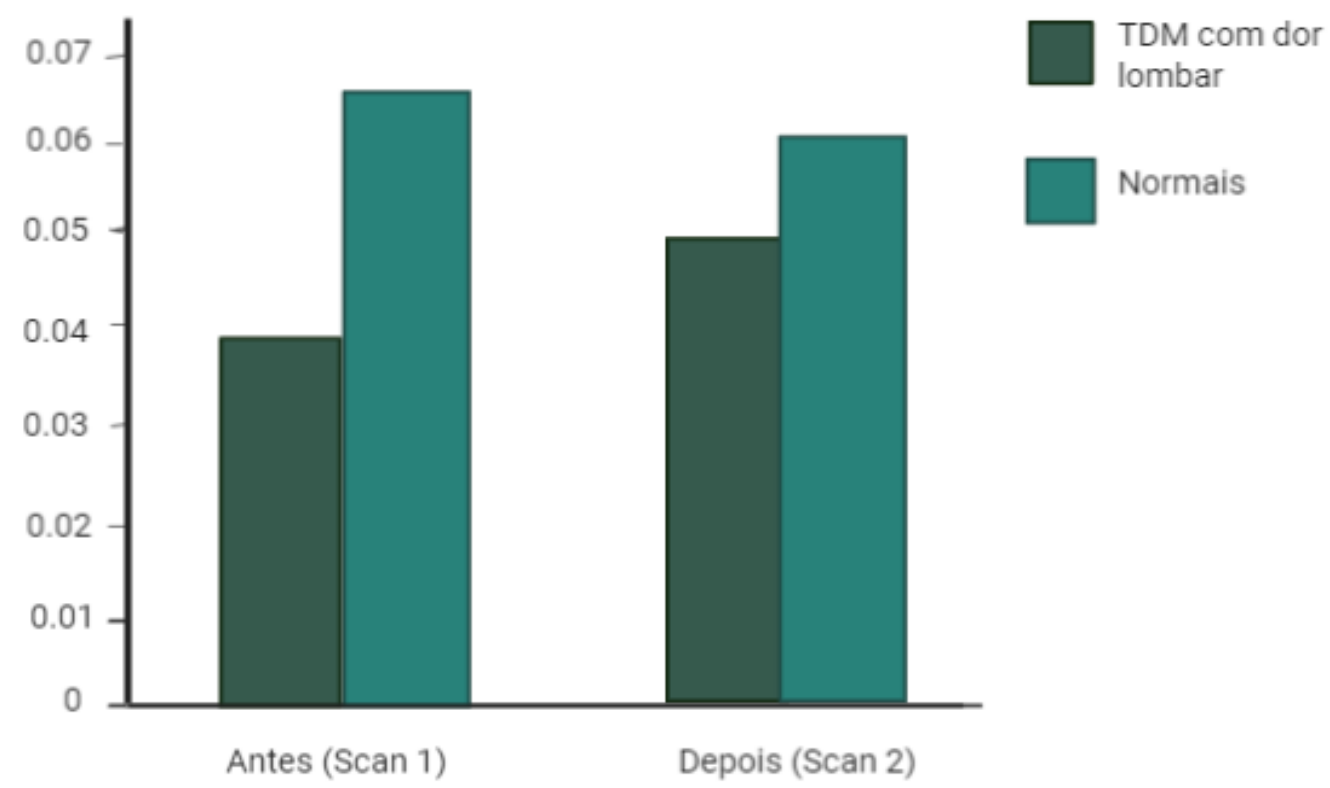

Figura 2. Níveis médios de GABA talâmico em sujeitos com Transtorno Depressivo Maior (TDM) e dor lombar $(n=2)$ comparados a sujeitos normais $(n=19)$ antes (Scan 1) e depois (Scan 2) de intervenção de Yoga de 12 semanas

Fonte: Streeter, Gerbarg, Saper, Ciraulo, Brown, 2012. ${ }^{20,}$ p. 573 


\section{Conclusão}

ISSN 2179-6750

O cenário imposto pela COVID-19 exigiu inúmeras medidas de segurança por parte das autoridades públicas e da população. O isolamento social, nunca estendido por tanto tempo e simultaneamente em todo mundo, tornou-se uma questão essencial tanto para preservar a vida quanto para evitar a superlotação da rede mundial de saúde pública, especialmente a brasileira, que já se encontra colapsada pelo crescente número de hospitalizações.

Dessa forma, houve adaptações em diversos países, devido a todas as preocupações e mudanças de rotina na vida profissional e pessoal. A saúde física da coletividade se sobressaiu em relação à saúde mental individual, que passou a ser tratada como fator secundário na ordem de importância, principalmente sobre os meios como cada indivíduo enfrenta o distanciamento social.

Essa abordagem mais introspectiva volta-se ao cuidado com ambas as dimensões humanas: a física e a psicológica. Por meio destas, a prática de ioga procura reafirmar a capacidade humana de lidar com o caos externo, causado pela insegurança e pelas más notícias frequentes, a fim de encontrar o equilíbrio e a paz interior através da sintonização entre corpo e mente.

Situada dentre as PICs, constituintes da Atenção Primária à Saúde, a ioga demonstra ser uma ferramenta importante para ajudar a acalmar as emoções, através da movimentação do corpo e de seu alinhamento com a respiração consciente, o que possibilita alcançar um equilíbrio mental e físico. Por meio dela, os praticantes aprendem a se conhecer e a observar a própria respiração, respeitando seu limite físico e, subsequentemente, controlando melhor seus pensamentos.

Para tanto, é necessário um sentimento sincero para procurar e experimentar uma nova prática e, às vezes, por si só. Por este motivo, aulas remotas com um professor ou amigo experiente que possa tirar as dúvidas, no início, pode ajudar muito a criar uma boa base. A ideia é que, com o passar do tempo, seja possível a conquista de uma prática pessoal e de rotina diária.

Tendo em vista a multiplicidade de fatores correlacionados à ioga, esta se evidencia, portanto, como uma ponte para um conhecimento profundo sobre si mesmo, sendo um investimento cultural nestes tempos de incerteza. Além disso, os conflitos internos não podem ser diluídos em uma única aula, por este motivo, precisa ser um caminho longo, com diálogo, constância, confiança e honestidade. 


\section{Referências}

1. World Health Organization, Regional Office for Europe. Mental health and COVID-19. [c2020; citado 27 jun. 2020]. Disponível em: http://www.euro.who.int/en/healthtopics/health-emergencies/coronavirus-covid-19/novel-coronavirus-2019-ncovtechnical-guidance-OLD/coronavirus-disease-covid-19-outbreak-technical-guidanceeurope-OLD/mental-health-and-covid-19

2. Ornell F, Schuch JB, Sordi AO, Kessler FHP. "Pandemic fear" and COVID-19: mental health burden and strategies. Braz J Psychiatry. 2020;42(3):232-5. https://doi.org/10.1590/1516-4446-2020-0008.

3. Peeri NC, Shrestha N, Rahman MH, Zaki R, Tan Z, Bibi S, et al. The SARS, MERS and novel coronavirus (COVID-19) epidemics, the newest and biggest global health threats: what lessons have we learned? Int J Epidemiol. 2020;49(3):717-26. https://doi.org/10.1093/ije/dyaa033.

4. Malta $M$, Rimoin AW, Strathdee SA. The coronavirus 2019-nCoV epidemic: Is hindsight 20/20? EClinicalMedicine. 2020;20:100289. https://doi.org/10.1016/j.eclinm.2020.100289. eCollection 2020 Mar.

5. Adhikari SP, Meng S, Wu Y-J, Mao Y-P, Ye R-X, Wang Q-Z, et al. Epidemiology, causes, clinical manifestation and diagnosis, prevention and control of coronavirus disease (COVID-19) during the early outbreak period: a scoping review. Infect Dis Poverty. 2020;9(1):29. https://doi.org/10.1186/s40249-020-00646-x.

6. Shigemura J, Ursano RJ, Morganstein JC, Kurosawa M, Benedek DM. Public responses to the novel 2019 coronavirus (2019-nCoV) in Japan: mental health consequences and target populations. Psychiatry Clin Neurosci; 2020;74(4): 281-2. https://doi.org/10.1111/pcn.12988.

7. Xiang YT, Yang Y, Li W, Zhang L, Zhang Q, Cheung T, et al. Timely mental health care for the 2019 novel coronavirus outbreak is urgently needed. Lancet Psychiatry. 2020;7(3):228-9. https://doi.org/10.1016/S2215-0366(20)30046-8.

8. Sharp J. Coping with coronavirus anxiety. Harvard Health Blog. 2020 Mar 12 [citado 27 jun. 2020]. Disponível em:_https://www.health.harvard.edu/blog/coping-withcoronavirus-anxiety-2020031219183.

9. Ministério da Saúde (BR). Práticas Integrativas e Complementares (PICS): qua is são e para que servem. [s.d.] [citado 27 jun. 2020]. Disponível em: https://saude.gov.br/saudede-a-z/praticas-integrativas-e-complementares. 
10. Galvanese ATC, Barros NF, D'Oliveira AFPL. Contribuições e desafios das práticas corporais e meditativas à promoção da saúde na rede pública de atenção primária do Município de São Paulo, Brasil. Cad Saude Publica. 2017;33(12):e00122016. https://doi.org/10.1590/0102-311x00122016.

11. Governo do Estado do Rio Grande do Sul. Nota Técnica 02/2018. Yoga na Rede de Atenção à Saúde. [Porto Alegre]: Departamento de Ações em Saúde Política Estadual de Práticas Integrativas e Complementares; 5 jun. 2018 [citado 27 jun. 2020]. Disponível em:_https://atencaobasica.saude.rs.gov.br/upload/arquivos/201808/24173406-notatecnica-02-2018-yoga.pdf.

12. Barros NF, Siegel P, Moura SM, Cavalari TA, Silva LG, Furlanetti MR, et al. Yoga e promoção da saúde. Cienc Saude Colet. 2014;19(4):1305-14. https://doi.org/10.1590/1413-81232014194.01732013.

13. Brown C. A Bíblia do yoga: o livro definitivo em posturas de yoga. São Paulo: Pensamento; 2009.

14. Tillu G, Chaturvedi S, Chopra A, Patwardhan B. Public Health Approach of Ayurveda and Yoga for COVID-19 Prophylaxis. J Altern Complement Med. 2020;26(5):360-4. https://doi.org/10.1089/acm.2020.0129.

15. Morgan N, Irwin MR, Chung M, Wang C. The effects of mind-body therapies on the immune system: meta-analysis. PLoS One. 2014;9(7):e100903. https://doi.org/10.1371/journal.pone.0100903. eCollection 2014.

16. Nascimento MVN, Oliveira IF. As práticas integrativas e complementares grupais e sua inserção nos serviços de saúde da atenção básica. Estud Psicol (Natal). 2016;21(3):27281. http://dx.doi.org/10.5935/1678-4669.20160026.

17. Balasubramaniam M, Telles S, Doraiswamy PM. Yoga on our minds: a systematic review of yoga for neuropsychiatric disorders. Front Psychiatry. 2013;3:117. http://dx.doi.org/10.3389/fpsyt.2012.00117. eCollection 2012.

18. Keyloun KR, Hansen RN, Hepp Z, Gillard P, Thase ME, Devine EB. Adherence and persistence across antidepressant therapeutic classes: a retrospective claims analysis among insured US patients with major depressive disorder (MDD). CNS drugs. 2017;31(5):421-32. http://dx.doi.org/10.1007/s40263-017-0417-0.

19. Xiong G, Doraiswamy PM. Does meditation enhance cognition and brain plasticity?. Ann N Y Acad Sci.2009;1172(1):63-69. http://dx.doi.org/10.1196/annals.1393.002. 
20. Streeter CC, Gerbarg PL, Saper RB, Ciraulo DA, Brown RP. Effects of yoga on the autonomic nervous system, gamma-aminobutyric-acid, and allostasis in epilepsy, depression, and post-traumatic stress disorder. Med Hypotheses. 2012;78(5):571-9. http://dx.doi.org/10.1016/j.mehy.2012.01.021.

\section{Minicurrículo}

Narottam Sócrates Garcia Chumpitaz | ORCiD: 0000-0002-6808-2642

Discente do curso de Medicina da Universidade Federal de Roraima - UFRR, Boa Vista, RR, Brasil.

Emille Magalhães Neves Campos | ORCiD: 0000-0003-0036-2561

Discente do curso de Medicina da Universidade Federal de Roraima - UFRR, Boa Vista, RR, Brasil.

Jhon Andreo Almeida dos Santos | ORCiD: 0000-0003-0416-1998

Discente do curso de Medicina da Universidade Federal de Roraima - UFRR, Boa Vista, RR, Brasil.

Giovanni de Souza Mota | ORCiD: 0000-0002 -1321-5495

Discente do curso de Medicina da Universidade Federal de Roraima - UFRR, Boa Vista, RR, Brasil.

Francisco Carlos Carneiro da Silva | ORCiD: 0000-0002-2234-052X

Médico pela Universidade Federal de Roraima. Assistente em Saúde Sexual e Reprodutiva no Fundo de Populações das Nações Unidas (2019). Médico na organização do Médicos Sem Fronteiras (2019). Médico na Organização Internacional para as Migrações (2020).

Karine Kersting Puls | ORCiD: 0000-0001-5712-4774

Médica de Família e Comunidade em São Pedro da Serra - RS. Membro do WONCA Working Party on Rural Practice (RuralWONCA) Councile Embaixadora do Rural Seeds. Docente da Universidade de Caxias do Sul - UCS, Curso de Medicina, Campus-Sede, Caxias do Sul, RS, Brasil. 